\title{
Thermodynamic and Operational Properties of Heterogeneous Lyophobic Systems
}

\author{
V. A. Eroshenko ${ }^{1 *}$, Y.G. Grosu ${ }^{2}$ \\ National Technical University of Ukraine “KPI”, Department of Energy Saving and Energy Management, \\ Laboratory of Thermomolecular Energetics, Peremogu pr. 37, Kiev, Ukraine. \\ E-mail: ${ }^{1}$ eroshenko@kpi.ua, ${ }^{2}$ grosu.y@ukr.net
}

\begin{abstract}
The grounds for further fundamental study of heterogeneous lyophobic systems (HLS) under technical thermodynamics in order to create nontraditional high-performance thermo-mechanical devices (dampers, accumulators and energy converters) are presented. A number of unusual HLS properties have been analyzed in view of their practical use: nontypical for conventional working bodies (gas, vapor) compression/decompression endo-/exothermal effects; operational features under adiabatic and cycling conditions. Thermodynamic model describing HLS behavior under conditions that are useful for operational requirements of technical devices and systems is proposed.
\end{abstract}

Keywords: Adiabatic; capillary; energy transformation; lyophobic; surface tension; thermodynamics.

\section{Introduction}

"Lyophobic porous solid - liquid" interfaces utilization in order to transform, dissipate and store mechanical energy provides new opportunities for nontraditional energetics (Eroshenko 1992, 1987b). The new working bodies heterogeneous lyophobic systems ${ }^{1}$ (HLS) - generate interest among scientists due to many unusual and useful properties and characteristics (Bougeard and Smirnov 2006; Cailliez et al. 2008; Coiffard, Eroshenko, Grolier 2005; Denoyel, Beurroies, Lefevre 2004; Eroshenko and Aistov 1990; Eroshenko and Lazarev 2012; Han and Qiao 2007; Iwatsubo, Washio, Yano, Miyazaki 2008; Karbowiak, Paulin, Bellat 2010; Laouir, Luo, Tondeur, Cachot, Le Goff 2003; Liu, Zhao, Culligan, Qiao, Chen 2009; Martin et al. 2002; Qiao, Punyamurtula, Han 2006).

New features of HLS are caused by new mechanism of energy transformation: contrary to conventional working bodies (gas and vapor which are successfully used over two centuries) where molecular kinetic energy is dominant, HLS takes advantage of potential energy of molecular interactions. Among the distinctive and promising characteristics of HLS extremely high energy density per unit volume should be mentioned (Laouir et al. 2003), which is similar to thermodynamic compactness (TC) of thermal machine (Eroshenko 1981, 1996): $T C=\frac{\Delta W}{\Delta V \Delta T}\left(\frac{J}{m^{3} K}\right)$, where $\Delta W$ is the net work output during the cycle, $\Delta V$ and $\Delta T$ are the volume and temperature change during the "heat - work" transformation in this cycle. Obviously TC is proportionally correlated with thermal efficiency of the cycle. Best modern thermal machines (working body is gas) have the following TC values ${ }^{2}$ (next: the name of cycle inventor - TC value $\left.\left(\mathrm{J} / \mathrm{m}^{3} \mathrm{~K}\right)\right)$ : Stirling -375 , Joule-Brayton -210 , Otto-Beau de

\footnotetext{
1 This systems was also called "repulsive clathrates" (Eroshenko 1997; Eroshenko and Lazarev 2012)

${ }^{2}$ Detailed calculation and analysis of TC for existing cycles according to available construction materials and temperature ranges are available elsewhere (Eroshenko 1987b).
}

Rochas - 490, Diesel - 520, Trinkler - 420. In spite of great variety of approaches and design solutions, TC of modern thermal machines has almost reached their ceiling values. It is possible to reach TC value abut $600-700\left(\mathrm{~J} / \mathrm{m}^{3} \mathrm{~K}\right)$ using conventional working bodies, but it requires new constructive materials which are able to stay under much higher gas temperature and pressure. These kinds of materials with acceptable cost are so far unavailable. While new HLS-based cycle ${ }^{3}$ gives opportunity to build thermal machine with TC value much higher (1-2 order of magnitude) compared to conventional ones. This promotes radical fuel economy, constructive materials amount reduction and environment safety level increase, as explosion stroke in HLS-thermal machine takes place under ambient temperature. So HLS utilization brings up the new paradigm in energy transformation process: utilization of surface free energy in heterogeneous lyophobic nanosystems ("liquid and porous matrix, which is not wetted by this liquid"), where liquid surface tension is intensive parameter of the system and huge $\left(200-1000 \mathrm{~m}^{2}\right.$ per 1 gram of the matrix) "solid - liquid" interface is extensive one.

The detailed expertize of HLS-based thermal machines is presented in (Laouir et al. 2003), dissipation devises characteristics and design solutions are described in (Eroshenko 2007; Eroshenko 2001a; Eroshenko, Piatiletov, Coiffard, Stoudenets 2007; Iwatsubo et al. 2008; Suciu, Iwatsubo, Deki 2003) and HLS-accumulation process features are given in (Eroshenko, Regis, Soulard, Patarin 2002; Qiao et al. 2006).

The purpose of this article is further and deeper analysis of HLS thermal and mechanical properties and development of specific mathematical methods to describe them. Presented models are tested using experimental data previously obtained (Coiffard et al. 2005, Karbowiak et al. 2009, 2010). Note that developing model which allows to predict adiabatic properties of the system according to its isothermal ones is rather important issue, because most

\footnotetext{
${ }^{3}$ Initially new cycle has been described in (Eroshenko 1981a, 1982) and detailed expertise is given in (Laouir et al. 2003)
} 
theoretical and experimental investigations of thermodynamic and thermophysical properties of HLS are carried out on the basis of scientific equipment, that allows to register only isothermal (slow) compression decompression processes, and power equipment operation typically requires understanding and predicting working media behavior in adiabatic (fast) conditions. We also focus on defining the thermodynamic characteristics of HLS based on matrices with an arbitrary pore size distribution function.

\section{Heterogeneous lyophobic systems principles}

Figure 1 represents principle scheme of new working body - HLS based on a matrix with different size pores (pore radius $r=$ var ). The system consists of a capillaryporous matrix 1 (volume $V_{\mathrm{M}}=$ const) and liquid 2 $\left(V_{\mathrm{L}}=\right.$ const $)$ which does not wet this matrix. Hypothetical piston 3 can be displaced in the cylinder 4 . As matrix is lyophobic (contact angle $\theta>90^{\circ}$ ) it eliminates the spontaneous penetration of fluid in the pore space. Volume reduction of the system during its forced decompression is due to interface area development (liquid intrusion into the porous matrix space), and not due to HLS components deformation (in this work we consider that matrix and liquid are incompressible).

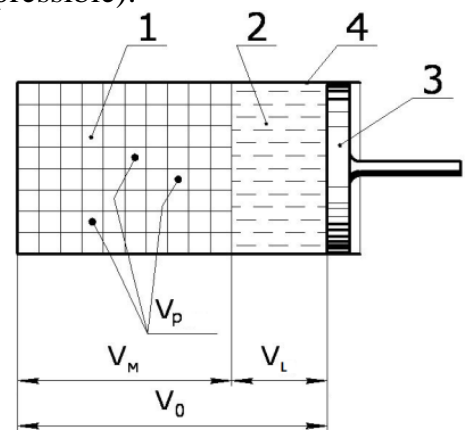

Figure 1. Heterogeneous lyophobic system physical model.

The HLS matrix can be presented as monolithic solid or as powder which porous particles are surrounded by fluid and have the same total volume of pore space as a monolithic solid. In the case of powder, the fluid volume should be increased according to interparticle volume, which must be filled during system compression. The following methodology is not affected by matrix nature (monolithic solid or powder).

\section{Approaches and assumptions for the correct HLS experimental results interpretation}

As system volume variation is only due to liquid penetration into the porous matrix space (interphase area development), it is usually defined as (Eroshenko 1990, 2007; Eroshenko and Grosu 2011):

$\mathrm{d} V=-k r \mathrm{~d} \Omega$

where $k$ is the pore space geometry coefficient $(k=0.50$ for long cylindrical capillary, $k=0.33$ for spheres).

Traditionally we express liquid intrusion pressure by the Laplace - Washburn equation (Washburn 1921), but in slightly modified form (with correcting factor B ):
$P_{\text {int }}=\frac{\sigma \cos \theta}{\mathrm{B}_{\mathrm{int}} \mathrm{k} r}$

where $\sigma$ is the "liquid - gas/vapor" surface tension (bulk phase). Here and below the $\cos \theta$ module is used and the value of contact angle $\theta$ is assumed to be taken for the planer "liquid - solid" interface.

Correcting factor B is used in equation (2) to preserve its original phenomenological structure despite the fact that microscopic properties of the liquid confined in the capillary, with diameter comparable with the size of the molecules of the liquid (Coiffard et al. 2005; Eroshenko 2000, 2001b; Karbowiak et al. 2009, 2010), may not coincide with its macroscopic properties, which are usually given for a massive phase in the references (Vargaftik 1972). Particularly, due to the molecular bonds number decrease (molecules were "wrested" from the bulk liquid phase and placed in a microcapillary), the liquid surface tension in such capillary is lower than in the bulk phase (Rusanov 1981, Kirkwood and Buff 1949), which is confirmed by molecular dynamic simulations according to which a single- or double-chain of liquid molecules is formed (Mann and Halls 2003; Moravsky and Loutfy 2004). Whereas, in water intrusion experiments on superhydrophobic silicalites (Coiffard et al. 2005; Eroshenko 2000, 2001b; Karbowiak et al. 2009, 2010) with the pore radius $r \sim 0,25 \mathrm{~nm}$ measured Laplace capillary pressure was about 5-7 times lower than calculated on the basis that confined and bulk water surface tensions are equal (Eroshenko 2002). Correction factor $B$ may be useful for more accurate calculation of non-standard processes in heterogeneous nanosystems, like HLS thermal effects of compression (intrusion) and decompression (extrusion).

In view of the above, the pressure of spontaneous extrusion of fluid from the pore space of the matrix will be determined by equation similar to Eq.(2):

$$
P_{\mathrm{ext}}=\frac{\sigma \cos \theta}{\mathrm{B}_{\mathrm{ext}} \mathrm{kr}}
$$

The difference between the values of $\mathrm{B}_{\text {int }}$ and $\mathrm{B}_{\text {ext }}$ reflexes experimentally observed hysteresis in HLS forced compression - spontaneous decompression cycle (Figure 2a, 2b: intruded water volume - pressure dependence. Dots represent experimental results obtained by Coiffard et al. 2005). Currently, the nature of this hysteresis is not fully understood, but there are a number of alleged reasons for this: the contact angle hysteresis (Coiffard and Eroshenko 2006), the presence of metastable states (Woo and Monson 2003), etc. Correcting factor $B$ is determined from experimental intrusion/extrusion pressure $\mathrm{P}_{\mathrm{int} \text {,ext }}^{*}$ and the one calculated according to original Laplace - Washburn equation ratio:

$$
\mathrm{B}_{\mathrm{int}, \mathrm{ext}}=\frac{\sigma \cos \theta}{\mathrm{P}_{\mathrm{int}, \mathrm{ext}}^{*} \mathrm{kr}}
$$


Numerous experimental results (Coiffard and Eroshenko 2006; Kong and Qiao 2005; Liu et al. 2009; Qiao et al. 2006) show that in most cases, the HLS pressure temperature dependence is nearly linear, which in view of Eqs. (2) and (3) allows to write it in the general form: $\sigma \cos \theta=A\left(T-T_{0}\right)+P_{0}^{\text {int,ext }}{ }^{\mathrm{k}} r_{0} \mathrm{~B}_{\text {int,ext }}$, where $A=\frac{\partial(\sigma \cos \theta)}{\partial T}$, $P_{0}^{\mathrm{int}, \text { ext }}$ is the intrusion/extrusion pressure at $T=T_{0}$ (initial temperature) under which pores with radius $r_{0}$ are filled.

The entropy of a heterogeneous system is the sum of its bulk and surface components (Guggenheim 1965), so that its variation can be expressed as:

$$
\mathrm{d} S_{\text {int,ext }}=\mathrm{d} S_{V}+\mathrm{d} S_{\Omega}=\frac{c v m}{T} \mathrm{~d} T+\frac{A}{\mathrm{~B}_{\text {int,ext }}} \mathrm{d} \Omega
$$

where $c_{V}$ is the HLS total specific thermal capacity at constant volume and $m$ is its mass.

We can see from Eq. (5) that for lyophobic systems $(A>0) \quad$ surface development $\left(\mathrm{d} \Omega>0, \mathrm{~B}_{\mathrm{int}}\right) \quad$ is accompanied by system temperature decrease (experimentally verified (Eroshenko 1987a)):

$$
\mathrm{d} S=0 \Rightarrow \mathrm{d} T_{\text {int,ext }}=-\frac{T A}{\mathrm{~B}_{\text {int,ext }} m c v} \mathrm{~d} \Omega
$$

One of the key characteristic of the $r=$ var porous matrix is its pore size distribution function - system volume variation as a function of radius of pores that are filled $\Gamma(r)=d V / d r$

PV-compression/decor the engineering stage this characteristic of the matrix rather important and is always known, so it is appropriate to use it as input information for the model. In this paper, we use slightly modified Gaussian function as approximation for $\Gamma(r)$ (Abramowitz and Stegun 1972):

$$
\Gamma(r)=\frac{d V}{d r}=\frac{r \mathrm{~b}}{\mathrm{a} \sqrt{2 \pi}} \exp \left(-\frac{\left(r-r_{0}\right)^{2}}{2 \mathrm{a}^{2}}\right),
$$

where $\mathrm{a}$ is the radius value deviation parameter and $b$ is the normalization factor. They are determined from the PVcompression isotherms of HLS (Figure 2).

\section{Specificity of HLS thermomechanical processes under isothermal and adiabatic conditions}

Isothermal conditions. We define the heat of isothermal interphase area development based on the classical thermodynamic concepts, but in view of the abovedescribed HLS specific: using traditional heat definition $\delta Q=T d S$

and Eq. (5) and considering the isothermal process $\quad\left(T=T_{0}=\right.$ const $)$, we obtain:

$\delta Q_{\text {int,ext }}=\mathrm{B}_{\text {int,ext }}^{-1} T_{0} A \mathrm{~d} \Omega$

The sign of the heat effect in Eg. (8) is determined according to the sign of the surface differential. Similarly, the expression for the work, defined as $\delta W=-P d V$, can be obtained by using Eq. (1) and (2): $\delta W_{\text {int,ext }}=\mathrm{B}_{\text {int,ext }}^{-1} \sigma \cos \theta \mathrm{d} \Omega$

Note, since in real systems, there is a nonzero compressibility of the matrix and the liquid, initial conditions for Eqs. (8) and (9) will be $Q(\Omega=0)=Q_{0}$ and $W(\Omega=0)=W_{0} \quad\left(Q_{0}\right.$ and $W_{0}$ are respectively heat and work produced during the system compression before reaching the intrusion pressure (Eq.(2)).

Since the aim of this paper is to study the processes of intrusion - extrusion of the liquid in the pore space of the matrix, the model does not include well-known compression - decompression processes of quasi condense components of the system (liquid + matrix), which with no harm for the problems discussed simplifies the mathematical expressions and specifies practical recommendations .

It is convenient to express interphase surface value during the compression - decompression in terms of easily controllable parameter of the system - its pressure. Thus, using Eqs. (1), (2) and (7), we obtain:

$\Omega=-\frac{1}{\mathrm{k}} \int \frac{\Gamma(r)}{r} d r=\frac{1}{\mathrm{k}} \int \frac{\Gamma(P)}{P} d P$

As indicated below, the solution of Eq. (10), in most cases can be solved using special functions (Abramowitz and Stegun 1972) and thus the amount of heat and work under isothermal conditions as a function of the pressure in the system and, consequently, as the degree of its compression can be obtained.

Adiabatic conditions. Under adiabatic conditions, the calculation of the work required to compress the system would be complicated due to the temperature decrease during interface $\Omega$ development (see Eq. (6)). This decrease is due to the fact that in isolated system interphase area development heat (8) is compensated not by environment heat supply (as it is under isothermal conditions), but by reducing internal energy of the system (its bulk phase) (Eroshenko 1987a). Solving the differential equation (6) and taking into account that initial temperature of the system is $T_{0}$, we obtain:

$T_{\text {int }}=T_{0} \exp \left(-\frac{\Omega A}{\mathrm{~B}_{\text {int }} m c v}\right)$

Initial conditions for the spontaneous decompression of the system are: $T_{0} \rightarrow \min \left(T_{\mathrm{int}}\right) \equiv T_{\mathrm{int}}^{\min }$-temperature reached after full HLS compression ( $\Omega=\Omega^{\max }$ ). Therefore, we get:

$T_{\text {ext }}=T_{\mathrm{int}}^{\min } \exp \left(\frac{\left(\Omega^{\mathrm{max}}-\Omega\right) A}{\mathrm{~B}_{\mathrm{ext}} m c_{V}}\right)$

To calculate HLS compression/decompression work under adiabatic conditions first we use its classical P-V coordinates definition and then take into account Eqs. (11), $(12),(1)-(4)$, and, $\sigma \cos \theta$ linear temperature dependence: 
$W_{\mathrm{int}}^{S}=-\int P d V=\mathrm{B}_{\mathrm{int}}^{-1} \int \sigma \cos \theta d \Omega=$

$=\left(\frac{A T_{0}}{\mathrm{~B}_{\mathrm{int}}}+P_{0}^{\mathrm{int}} \mathrm{k} r_{0}\right) \Omega+T_{0} m c v\left(\exp \left\{-\frac{\Omega A}{\mathrm{~B}_{\mathrm{int}} m c v}\right\}-1\right)$

$W_{\mathrm{ext}}^{S}=-\left(\frac{A T_{0}}{\mathrm{~B}_{\mathrm{ext}}}+P_{0}^{\mathrm{ext}} \mathrm{k} r_{0}\right)\left(\Omega_{\text {max }}-\Omega\right)+$

$+T_{\mathrm{int}}^{\mathrm{min}} m c v\left(\exp \left\{\frac{\left(\Omega_{\mathrm{max}}-\Omega\right) A}{\mathrm{~B}_{\mathrm{ext}} m c v}\right\}-1\right)$

Taking into account the pressure-temperature dependence under adiabatic conditions in Eq. (10), equations (10) - (14) allow us to calculate system work and its temperature at any stage of HLS compression and decompression.

\section{Proposed model validation}

Verification of proposed model adequacy was performed using calorimetric and volumetric data (experiment procedure described in (Coiffard et al. 2005; Karbowiak et al. 2009, 2010)). Experiments were obtained on three heterogeneous lyophobic systems based on superhydrophobic silicalites with quasi-cylindrical pores (average radius $r_{0} \sim 0,25 \mathrm{~nm}$ ).

In all systems, the working fluid is distilled water, and the difference between systems comes from matrix synthesis mode: for MFI-OH matrix reaction medium contained $\mathrm{OH}^{-}$ions (Coiffard et al. 2005), while for MFIF1 (Coiffard et al. 2005) and MFI-F2 (Karbowiak et al. 2009, 2010) matrices $\mathrm{F}^{-}$ions. These features led to a different hydrophobicity of the matrices. Thus, the use of fluoride anions instead of hydroxide, apparently, leads to a number of lattice defects decrease and hence a smaller number of hydrophilic silanol groups. This leads to a greater hydrophobicity of the MFI-F1 and MFI-F2 matrices and, consequently, according to Eq. (2) to the increasing intrusion/extrusion pressure, which is reflected on the experimental compression/decompression PV-isotherms (see Figure 2).

Using Eqs. (2), (7) and compression PV-isotherms (Figure 2), we obtain $\Gamma(r)$ function for the matrices (Figure 2 frame). The approach we used to calculate thermal effects and work as an experimental input information requires $\mathrm{PV}$-compression/decompression isotherms, as they allow to obtain $^{\Gamma(r)}$ function (7), which gives us developed "liquid solid" interface value at every stage of the system compression/decompression and hence its heat and work.

Approximating the experimental $d V / d r$ dependence (Figure 2, frame), calculated from the PV-compression isotherms, we obtain for the MFI-F1:

$$
\begin{aligned}
& \mathrm{b}_{\mathrm{MFI-F} 1}=397,5, a_{\mathrm{MFI-F} 1}=2 \cdot 10^{-3}(\mathrm{~nm}) ; \mathrm{MFI}-\mathrm{F} 2: \\
& \mathrm{b}_{\mathrm{MFI-F} 2}=370, a_{\mathrm{MFI-F} 2}=3 \cdot 10^{-3}(\mathrm{~nm}) ; \mathrm{MFI}-\mathrm{OH}: \mathrm{b}_{\mathrm{MFI-OH}}=215, \\
& a_{\mathrm{MFI-OH}}=4 \cdot 10^{-3}(\mathrm{~nm}) .
\end{aligned}
$$

Despite the fact that described matrices are defined as mono-porous ( $r=$ const $)$, a small pressure dispersion during the intrusion process is experimentally observed for all the samples (Figure 2), which under the proposed approach is reflected as non-zero $a_{\mathrm{MFI-F1}}, a_{\mathrm{MFI-F} 2}$ and $a_{\mathrm{MFI}-\mathrm{OH}}$ values. Such small variations in the pressure of the intrusion can hardly be caused by the presence of different pore radii in the matrices. However, as it is shown below, this phenomenon account is necessary, and the use of nonzero radius value deviation parameter for this matter is acceptable.

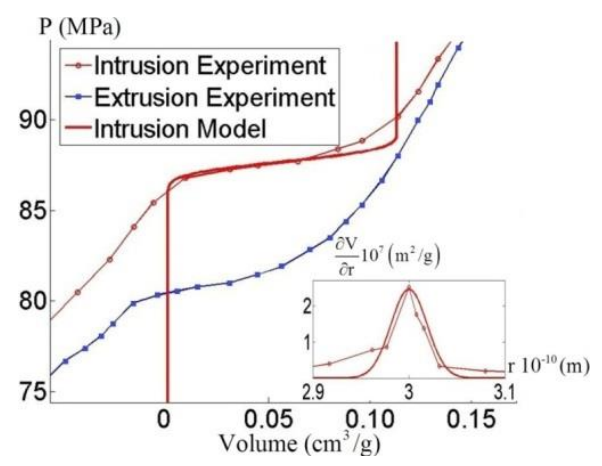

Figure 2a. Isothermal (303K) PV diagram during a cycle for MFI-FI + water and its $\Gamma(r)$ function in the frame

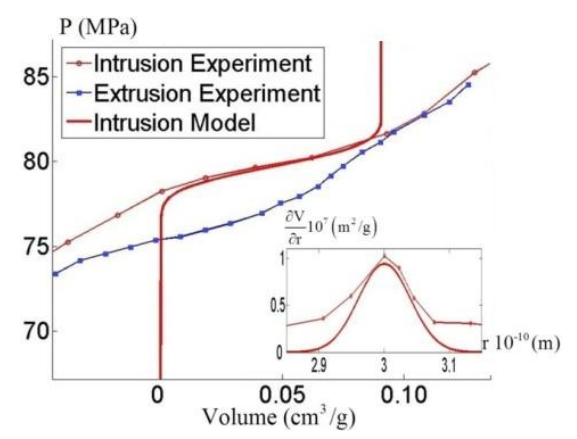

Figure 2b. Isothermal (303K) PV diagram during a cycle for $\mathrm{MFI}-\mathrm{OH}+$ water and its $\Gamma(r)$ function in the frame

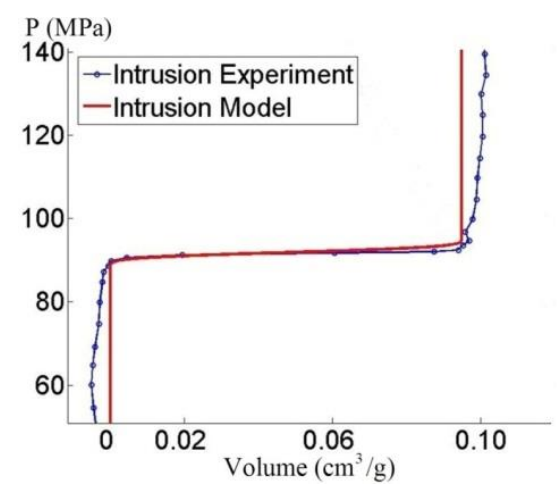

Figure 2c. Isothermal (298K) PV diagram during intrusion for MFI-F2 + water

Also note, that it is possible to avoid minor discrepancies of experimental and theoretical curves (Figure 2), using a more complex distribution function than Eq.(7), but that unnecessarily increases calculations cumbersome.

Nevertheless, the use of the approximate function (7) still gives a quite satisfactory outcome prediction of thermal effects: in worst case scenario of MFI-OH based system the experimental intrusion heat effect is $5.7 \pm 0.2(\mathrm{~J} / \mathrm{g})$ versus calculated value of $6.14(\mathrm{~J} / \mathrm{g})$, which gives a relative error not exceeding $\sim 10 \%$. 
Using Eq. (1), (7) and (10), interface value may be expressed as:

$\Omega=-\frac{\mathrm{b}}{2 \mathrm{k}}\left(\operatorname{erf}\left(\frac{r-r_{0}}{\sqrt{2} \mathrm{a}}\right)-\operatorname{erf}\left(\frac{r_{\max }-r_{0}}{\sqrt{2} \mathrm{a}}\right)\right)$

where $\operatorname{erf}(x)=\frac{2}{\sqrt{\pi}} \int_{0}^{x} \exp \left(-\mathrm{t}^{2}\right) \mathrm{dt}$ is the error function (Abramowitz and Stegun 1972), and $r_{\max }$ is conditional maximum pore radius of the matrix, at which intrusion begins $(\Omega \approx 0)$.

In the following calculations we use the assumption " $\theta \approx$ const", which is correct for a number of HLS (Naidich 1972), while for others HLS, the contact angle might be slightly dependent on the temperature (Coiffard and Eroshenko 2006). In view of MFI matrices superhydrophobicity (Coiffard et al. 2005; Karbowiak et al. 2009, 2010) $\operatorname{consider} \cos \theta \approx 1$. Keeping in mind that experimental $\mathrm{PV}$-compression/decompression dependence is input parameter of the model, coefficient $B_{\text {int,ext }}$ approximation compensates inaccuracies associated with contact angle value. Using Eq. (2) - (4) we obtain for system based on a MFI-F1 matrix $\left(T_{0}=303 \mathrm{~K}, \quad \mathrm{~d} \sigma / \mathrm{d} T=-0,16^{*} 10^{-3}\left(\mathrm{Hm}^{-1} \mathrm{~K}^{-1}\right)\right)$ : $\mathrm{B}_{\mathrm{int}} \approx 5,41, \quad \mathrm{~B}_{\mathrm{ext}} \approx 5,93 ;$ for MFI-F2: $\mathrm{B}_{\mathrm{int}} \approx 5,68$, $\mathrm{B}_{\mathrm{ext}} \approx 6,48$; for MFI-OH: $\mathrm{B}_{\mathrm{int}} \approx 5,88, \mathrm{~B}_{\mathrm{ext}} \approx 6,25$. Next, using Eqs. (1) - (4), (7) and (8), heat flow per pressure variation $\Phi_{\mathrm{P}}$ can be expressed as:

$\Phi_{\mathrm{P}}^{\mathrm{int}, \mathrm{ext}}=\frac{\mathrm{d} Q}{\mathrm{~d} P}= \pm \Gamma(r) r \frac{T_{0} B_{\text {int,ext }}}{\sigma} \frac{\mathrm{d} \sigma}{\mathrm{d} T}= \pm \frac{\Gamma(P) T_{0}}{P_{\text {int,ext }} k} \frac{\mathrm{d} \sigma}{\mathrm{d} T}$

here "plus" sign refers to the process of intrusion, and "minus" - to extrusion.

The compressibility of the system (volume variation per pressure variation) can be written as:

$\varepsilon_{\text {int,ext }}=\frac{\mathrm{d} V}{\mathrm{~d} P}=-\frac{\mathrm{k} r^{2} \Gamma(r)}{\sigma B_{\text {int,ext }}}$

Figure 3 represents theoretical and experimental (Coiffard et al. 2005; Karbowiak et al. 2009, 2010) results comparison of $\Phi_{\mathrm{P}}$ pressure dependence. To put in correspondence thermal and mechanical processes of HLS, both theoretical and experimental compressibility of the system $\varepsilon_{\text {int }}$, as a function of pressure, is shown in the same Figure (bottom frame).

As might be seen, there is a reasonable agreement between theoretical and experimental curves for the liquid intrusion and extrusion processes followed by respectively endo- and exothermal effects. For the compression processes it is shown that maximum end thermal effect variation coincides with the maximum system compressibility (Figure 3 bottom frame). Figure $3 a$ top frame also shows experimental and theoretical (Eq. (8) and (15)) heat of interphase area development as a function of pressure for "MFI-F1 + water" system. Experimental and model compressibility curves correlation (Figure 3, bottom frame) shows that this parameter can also be predicted within approach proposed. $\Phi_{\mathrm{P}}$ and $\varepsilon$ curves comparison confirms the fact that the greatest system heat demand appears at the maximum interface development trends $\mathrm{d} \Omega / \mathrm{d} P$

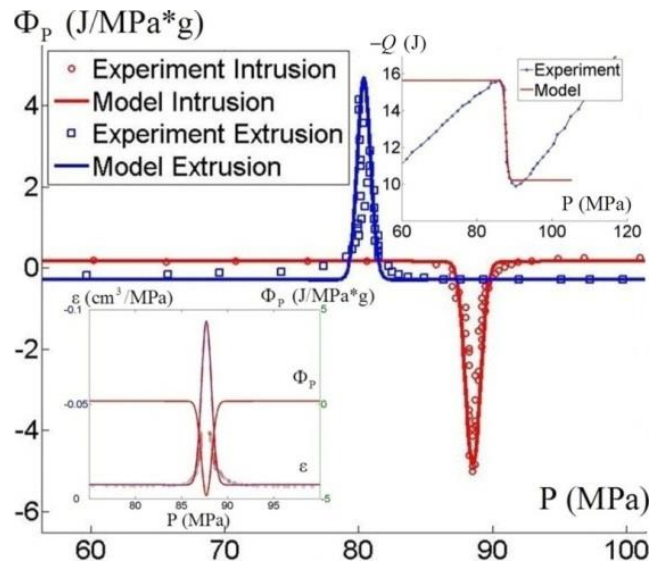

Figure 3a. MFI-F1 + water cycle $(303 K)$ : Heat flow $\Phi_{\mathrm{P}} v$ s. pressure. $\Phi_{\mathrm{P}}$ and compressibility $\varepsilon$ comparison (bottom frame). Thermal energy $-Q$ during compression (top frame)

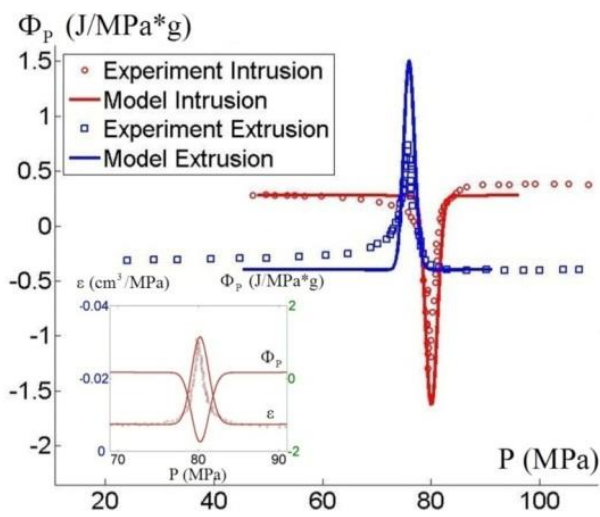

Figure $3 b . \mathrm{MFI}-\mathrm{OH}+$ water cycle $(303 \mathrm{~K})$ : Heat flow $\Phi_{\mathrm{P}}$ vs. pressure. $\Phi_{\mathrm{P}}$ and compressibility $\varepsilon$ comparison (frame)

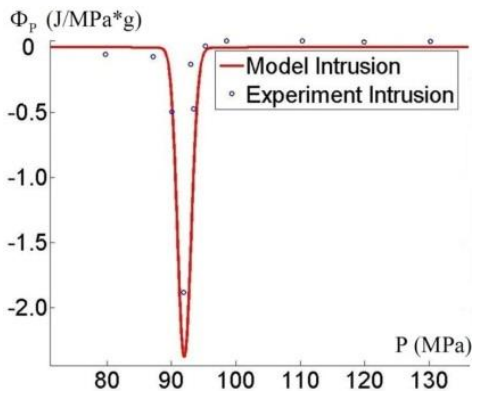

Figure 3c. MFI-F1 + water cycle $(298 K)$ : Heat flow $\Phi_{\mathrm{P}} v s$. pressure.

As mentioned above, described approach allows predicting behavior of the system under adiabatic conditions. Figure 4 shows intrusion work - pressure dependence comparison in a heterogeneous system «MFIF1 + Water" (Coiffard et al. 2005) under isothermal conditions (experiment and model ((9), (15)) based on the incompressible component of the system) and under adiabatic conditions after 150 and 300 successive 
intrusion/extrusion cycles (HLS successive cycling is described below).

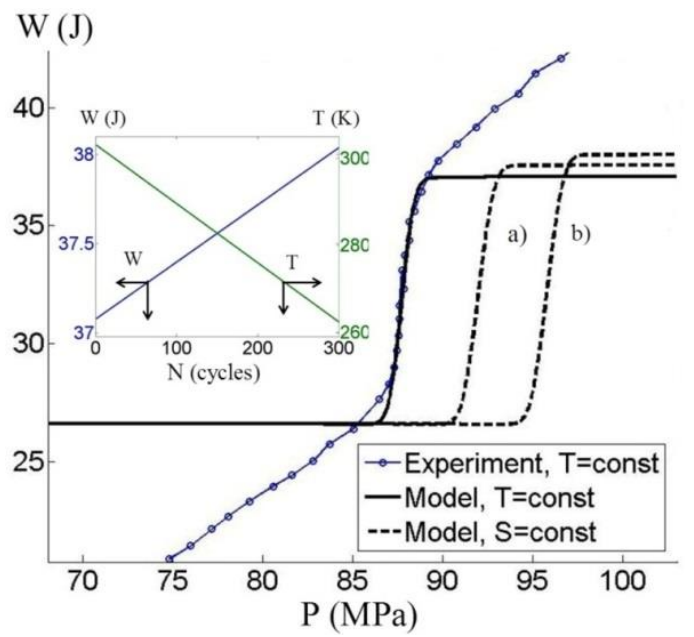

Figure 4. Mechanical energy vs. pressure during the compression of MFI-F1 + water under isothermal (303K) and adiabatic conditions after a) 150 and b) 300 cycles.

As might be seen from Figure 4, there is good agreement between simulated and experimental curves under isothermal conditions, which confirms the adequacy of the thermodynamic description of the HLS based on porous matrix with pore radius in angstrom range.

According to Eq. (11) temperature decrease, due to full HLS compression ( $\Omega=\Omega_{\max }$ ) under adiabatic conditions, reaches $\Delta T_{\text {int }} \approx 1,5(\mathrm{~K})$, which increases the work expended in system compression on the value of $\Delta W \approx 20(\mathrm{~mJ})$. Although, this increase is rather small in comparison with the work of compression under isothermal conditions $W \approx 10^{4}(\mathrm{~mJ})$, its contribution begins to play a significant role in high-cycle system loading. The explanation of this effect should be given: experiments designed to study the temperature dependence of HLS pressure, show this dependence is different for intrusion and extrusion processes (Kong and Qiao 2005; Liu et al. 2009; Qiao et al. 2006): $\frac{\mathrm{d} P_{\text {int }}}{\mathrm{d} T}>\frac{\mathrm{d} P_{\text {ext }}}{\mathrm{d} T}$ (which is reflected in described approach as $\mathrm{B}_{\mathrm{int}}<\mathrm{B}_{\mathrm{ext}}$ ), and as it follows from Eqs. (11), (12) and (2), (3) leads to a consistent decrease in temperature of the system after each cycle of the "forced compression - spontaneous decompression" under adiabatic conditions: $\max \left(T_{\mathrm{ext}}\right)<T_{0}$.

As might be seen from Figure 4, this temperature reduction leads to an increase of intrusion-extrusion pressure (according to Eqs. (2) - (3)) and, consequently, to an increase of the work expended for system compression. The same Figure (frame) displays $W^{S}$ (system compression work) and $T$ (temperature) at the end of each next successive intrusion/extrusion cycle, as a function of the number of the cycles $N$.

As might be seen, there are linear relationships: $W^{S}$ growth and $T$ decline as number of cycles $N$ increase. So, after 100 adiabatic cycles growth is $\Delta W \sim 330(\mathrm{~mJ})$, and the temperature drop is $\sim 15(\mathrm{~K})$.

\section{Discussion}

Presented in Figure 3 data suggest that thermal effects, due to the compression-decompression of heterogeneous lyophobic systems based on capillary-porous matrices with radii in the nanometer range, are associated with significant energy cost/release during the development/ reduction of the huge interface (breaking of intermolecular bonds during intrusion of the liquid is accompanied by endothermal effect, and their recovery - by exothermal effect).

As might be seen from Figure 3, the peak of the system heat flow is observed during isothermal filling of pores with

radii close to $r_{0}$, the value that indicates the maximum heat demand must be satisfied at the moment of maximum interface development $\Omega$ to meet the isothermal conditions. This property, in the present model and experiments carried out by (Coiffard et al. 2005 and Karbowiak et al. 2009, 2010), suggests that compression mechanical work is greater under adiabatic conditions, due to system temperature drop (6). This phenomenon has been observed experimentally (Eroshenko 1987a); abnormally high intrusion pressure rise, due to velocity of the HLS compression increase, was apparently because of the fact that under quasiadiabatic conditions (stroke!) the interface development heat was taken only from that part of the system, which is directly adjacent to the mouth of the pore (channel), apparently due to the thermal resistance of the medium. Occurring local anomalous temperature drop near the pore mouth led to a substantial liquid surface tension increase in that area, and therefore, to local capillary Laplace pressure increase (2), (pressure of the intrusion), and hence to a whole heterogeneous system pressure increase (Pascal's hydraulic law).

In view of Eq. (9) and linear $\sigma \cos \theta$ temperature dependence, the difference between the values of work under adiabatic and isothermal conditions can be displayed as:

$$
\begin{aligned}
& \delta W_{\text {int }}^{S}=\left.\delta W_{\text {int }}\right|_{T=T_{0}}+\mathrm{B}_{\text {int }}^{-1}\left(T_{0}-T_{\text {int }}\right) A \mathrm{~d} \Omega \\
& \delta W_{\text {ext }}^{S}=\left.\delta W_{\text {ext }}\right|_{T=T_{\text {int }}^{\text {min }}}+\mathrm{B}_{\text {ext }}^{-1}\left(T_{\text {int }}^{\text {min }}-T_{\text {ext }}\right) A \mathrm{~d} \Omega
\end{aligned}
$$

Since during intrusion $\delta W_{\text {int }}>0$ (work is done on the system), $T_{0}-T_{\text {int }}>0$ (according to (11), temperature decreases during intrusion), and $d \Omega>0$ (development of the interface), adiabatic compression work is greater than isothermal one on the value of $\Delta W_{\mathrm{int}}=\mathrm{B}_{\mathrm{int}}^{-1}\left(T_{0}-T_{\mathrm{int}}^{\mathrm{min}}\right) A \Omega_{\max }$ . Using the same logic for extrusion process $(d \Omega<0$, $\left.\delta W_{\text {ext }}<0, \quad\left(T_{\mathrm{int}}^{\min }-T_{\mathrm{ext}}\right)<0\right) \quad$ it $\quad$ is obtained $\Delta W_{\text {ext }}=\mathrm{B}_{\text {ext }}^{-1}\left(T_{\mathrm{int}}^{\min }-T_{\mathrm{ext}}^{\max }\right) A \Omega_{\max }$. Due to the temperature irreversibility during intrusion-extrusion cycle under adiabatic conditions inequation $T_{0}>T_{\mathrm{ext}}^{\mathrm{max}}$ can be obtained, and taking into account the fact that $\mathrm{B}_{\mathrm{ext}}>\mathrm{B}_{\mathrm{int}}$, it could be shown that $\Delta W_{\text {ext }}<\Delta W_{\text {int }}$. Such system reaction on cycling leads to continuous growth of the difference between the compression and decompression work, with each successive adiabatic cycle. In other words dissipative power of the system will increase (intrusion-extrusion pressure hysteresis increase) 
Note that using Eqs. (8) and (9), equations (13) and (14) might be rewritten as

$$
\begin{aligned}
& W_{\mathrm{int}}^{S}=W_{\mathrm{int}}+\left|Q_{\mathrm{int}}\right|-m c_{V}\left(T_{0}-T_{\mathrm{int}}\right), \\
& W_{\mathrm{ext}}^{S}=W_{\mathrm{ext}}-\left|Q_{\mathrm{ext}}\right|+m c_{V}\left(T_{\mathrm{ext}}-T_{\mathrm{int}}^{\mathrm{min}}\right) .
\end{aligned}
$$

According to Eq. (18) the difference $Q_{\text {int }}-m c_{V}\left(T_{0}-T_{\text {int }}\right)>0$, so the work of intrusion in adiabatic process will always be greater than in isothermal one. For the process of spontaneous adiabatic extrusion of the system (21) this effect is converse (adiabatic operation will be less then isothermal one) according to the inequality: $-\left|Q_{\mathrm{ext}}\right|+m c_{V}\left(T_{\mathrm{ext}}-T_{\mathrm{int}}^{\mathrm{min}}\right)<0$ (see Eq. (19)).

Equations (20) and (21) show that work under adiabatic conditions can be expressed as an algebraic sum: conditional work of the interphase area development/reduction under isothermal conditions $W_{\text {int,ext }}$ at $T=T_{0}$ (9) plus a conditional heat of the interface development/reduction under isothermal conditions $Q_{\text {int,ext }}$ at $T=T_{0}$ (8), which is compensated by reducing the internal energy of the system, (its temperature) - third term. Note, that on the basis of equations (13) and (14), adiabatic work of compression/decompression is structurally identical to the same work obtained previously for lyophilic systems (Laouir and Tondeur 2008), which indicates the absence of contradictions of proposed approach with the energy balance equations and correct supplement of practically useful Equations (13) and (14).

A nontrivial property of the system to reduce its temperature after each subsequent adiabatic cycle of compression / decompression (Figure 4b), can be effectively used to create a fundamentally new class of refrigerators. High-pressure cold generating process based on matrices with $r<1(\mathrm{~nm})$ provides an extremely high thermodynamic compactness (Eroshenko 1981a, 1996) ( $\delta W_{\mathrm{C}} /(\Delta V \Delta T)$ ) of thermomolecular coolers (cooling capacity $W_{\mathrm{C}}$ per volume unit of the working chamber and per one degree of working temperature range).

Taking into account HLS temperature reduction, due to its adiabatic cycling, is no less important in case of dissipating systems creating, because this effect will lead to an increase of intrusion pressure (2) and the work of compression (13), which increases the resistance of the HLS to external mechanical disturbances.

An additional advantage of this approach is its applicability for the description of the systems based on matrices with varying complexity pores radii distribution function $\Gamma(r)$ (including composites based on several different matrices). In this case $\Gamma(r)=\sum_{i} \Gamma_{i}(r)$, where $\Gamma_{i}(r)$ is the pore radii distribution function of $i$-th porous matrix, and the description logic remains unchanged. It is important to note, that a purely thermodynamic approach to describing the behavior of these systems is justified only for samples that are based on matrices with ultra-low pore radius $(r-1 \mathrm{~nm}$ and lower), since when $r>1 \mathrm{~nm}$ the system shows a more complex nature of

dependencies studied in this work (Coiffard et al. 2005; Karbowiak et al. 2009, 2010) and for an adequate description consideration of complex mechanics of the process seems to require. In-depth understanding of these processes with the further extension of the studied systems class is the subject of future studies.

\section{Conclusions}

1. Theoretical and experimental analysis of heterogeneous lyophobic systems (HLS) thermodynamic and operational properties allows, using as an input experimentally easy determent $\mathrm{P}-\mathrm{V}$ isotherm, to understand the nature of some HLS phenomena and to predict practically useful effects and properties on every compression/decompression stage:

- Nontraditional thermal responses due to system mechanical perturbation and relaxation. - Temperature variations due to adiabatic compression / decompression.

- Compression / decompression work under isothermal and adiabatic conditions and a comparison of these works.

- Compressibility reduction of the system as it becomes compressed in adiabatic process (Eroshenko 1987a, 2007; Eroshenko et al. 2007).

2. Theoretically confirmed the experimentally observed (Eroshenko 1987a) temperature drop of the system after intrusion/extrusion cycle under adiabatic conditions.

3. Theoretically predicted and experimentally observed properties of heterogeneous lyophobic systems based on different types of matrices ( $r=$ const or $r=$ var $)$ (Coiffard et al. 2005; Eroshenko 1987b, 2007; Eroshenko et al. 2007; Karbowiak et al. 2009, 2010), offer the prospect of creating a fundamentally new working mediums for nanotechnology (Eroshenko 1981a, 1996), cost-saving and ecological friendly systems and equipment in modern energetics (Eroshenko 1996, 1997, 2001a, 2007; Eroshenko et al. 2007; Han and Qiao 2007; Iwatsubo et al. 2008; Martin et al. 2002; Qiao et al. 2006).

\section{Nomenclature}

b

$\mathrm{B}$

C

C H

$\mathrm{k}$ $\mathrm{m}$ $\mathrm{N}$ P

$Q$ $\mathrm{RC}$ $r$ S $T$ V W pore radii dispersion parameter distribution function coefficient pressure correction coefficient heat capacity specific heat capacity

LS heterogeneous lyophobic system pore geometry coefficient mass

cycle number

pressure

heat

repulsive clathrate

pore radius

entropy

temperature

volume

work

pore radii distribution function

volume variation per pressure variation contact angle $\mathrm{m}$

$\mathrm{m}^{2}$

$\mathrm{JK}^{-1}$

$\mathrm{JK}^{-1} \mathrm{~kg}^{-1}$

$\mathrm{kg}$

$\mathrm{Pa}$

$\mathrm{J}$

$\mathrm{m}$

$\mathrm{JK}^{-1}$

K

$\mathrm{m}^{3}$

J

$\mathrm{m}^{2}$

$\mathrm{cm}^{3} \mathrm{~Pa}^{-1}$ 
$\Phi$ heat flow per pressure variation

$\Omega \quad$ surface area

\section{Subscripts}

\section{$\mathrm{P}$}

MFI-F1

MFI-F2

MFI-OH

int

ext

$*$

V

$\Omega$

$\mathrm{S}$

\section{Superscript}

$\begin{array}{ll}\min & \text { minimum } \\ \max & \text { maximum }\end{array}$

\section{References}

Abramowitz, M., and Stegun, I., A. (1972). Handbook of Mathematical Functions with Formulas, Graphs, and Mathematical Tables. New York: Dover.

Bougeard, D. and Smirnov, K., S. (2006) Modelling studies of water in crystalline nanoporous aluminosilicates. Physical Chemistry Chemical Physics. 9(2), 226-245.

Cailliez, F., Trzpit, M., Soulard, M., Demachy, I., Boutin, A., Patarin, J., and Fuchs, A., H. (2008). Thermodynamics of water intrusion in nanoporous hydrophobic solids. Physical Chemistry Chemical Physics, 10(32), 4817-4826.

Coiffard, L., Eroshenko, V. (2006). Temperature effect on water intrusion/expulsion on grafted silica gels. J. of Colloid and Interface Science. 300(1), 304-309.

Coiffard, L., Eroshenko, V., A., Grolier, J.-P., E. (2005). Thermomechanics of the Variation of Interfaces in Heterogeneous Lyophobic Systems. AIChE J, 51(4), 1246-1257.

Denoyel, R., Beurroies, I., Lefevre, B. (2004). Thermodynamics of wetting: information brought by microcalorimetry. J. of Petroleum Science \& Engineering, 45(3-4), 203-212.

Eroshenko V. A. and Lazarev Yu. F. (2012). Rheology and dynamics of repulsive clathrates. J. Of Applied Mechanics And Technical Physics, 53(1), 98-112.

Eroshenko, V. A. (1981a). Heterogeneous Thermodynamic System, Eroshenko Cycle of Transformation of Thermal Energy into Mechanical Energy and Devices to Achieve It (in Russian). Soviet-Russian Patent No. 1,254,811.

Eroshenko, V. A. (1987a). Effect of Heat Exchange on Filling of Lyophobic Pores and Capillaries with Liquid. Colloid J.USSR, 49, 769-773.

Eroshenko, V. A. (1987b). Limiting efficiency or maximum thermodynamic compactness of Heat Engines (in Russian). USSR Academy of Science J., Energy and Transport, 2, 125-133.
Eroshenko, V. A. (1990) Unusual Properties of One complex Thermodynamic System (in Russian). C. $R$. Acad. Sci. Ukraine, Ser. A, 10, 79.

Eroshenko, V. A. (1992). Thermomolecular energetics (in Russian). Industrial Heat Engineering, 14(1-3), 22-25.

Eroshenko, V. A. Heat Engines and Different Energy devices. Soviet-Russian Patents: No943,444 (1981b); No1,382,078 (1982); No1,380,357 (1983); No1,333,870 (1985); No1,434,881 (1985); No1,452,262 (1986); No1,508,665 (1987c).

Eroshenko, V. A., and Aistov, V. I. (1990). Heat Engine Cycles Optimization According to Thermodynamic Compactness (in Russian). Industrial Heat Engineering, 12(3), 60-64.

Eroshenko, V. A., Grosu, Y. G. (2011). Properties of Heterogeneous Lyophobic Systems Based on Matrixes With Different Pore Size (in Russian). Industrial Heat Engineering, 33(6), 73-79.

Eroshenko, V., A. (1996). Heterogeneous structure for accumulation or dissipation of energy, process to use it and associated devices. Int. Patent WO 96/18040.

Eroshenko, V., A. (1997). Repulsive clathrates. New operational material for efficient seismic isolation. Proceedings of International Post-SMITR Conference «Seismic Isolation, passive Energy Dissipation and active Control of Seismic Vibrations of Structure», Taormina, Sicily, Italy, August 25 to 27, 783-794.

Eroshenko, V., A. (2001a). Damper with high dissipating power. Int. Pat. WO 01/55616 A1, 1-33.

Eroshenko, V., A. (2007). A New Paradigm of Mechanical Energy Dissipation. Part 1: Theoretical Aspects and practical Solutions. Journal Proceedings of Mechanical Engineers, Part D: Journal of Automobile Engineering, 221(3), 285-300.

Eroshenko, V., A., Piatiletov, I., I., Coiffard, L., and Stoudenets, V., P. (2007). A New Paradigm of mechanical Energy Dissipation. Part 2: Experimental Investigation and effectiveness of a novel Damper. Journal Proceedings of Mechanical Engineers, Part D: Journal of Automobile Engineering, 221(3), 301-312.

Eroshenko, V., Regis, R.-C., Soulard, M., and Patarin, J. (2001b). Energetics: A New Field of Applications for Hydrophobic Zeolites. J. of American Chemical Society, 123(33), 8129-8130.

Eroshenko, V., Regis, R.-C., Soulard, M., and Patarin, J. (2002). The heterogeneous systems 'water-hydrophobic zeolites': new molecular springs. C.R.PHYSIQUE, 3(1), 111-119.

Guggenheim, E., A. (1965). Thermodynamique. Paris: «Dunod».

Han, A., and Qiao, Y. (2007). A volume-memory liquid. Applied Physics Letters, 91(17), 173132.

Iwatsubo, T., Washio, K., Yano, H., and Miyazaki, M. (2008). Experimental Study of a Colloidal Damper to Practical Application. J. of System Design and Dynamics, 2(5), 1160 - 1169. 
Karbowiak, T., Paulin, C., Ballandras, A., Weber, G., and Bellat, J.-P. (2009). Thermal Effects of Water Intrusion in Hydrophobic Nanoporous Materials. J. of American Chemical Society Communications, 131(29), 9898 9899.

Karbowiak, T., Paulin, C., Bellat, J.-P. (2010). Determination of water intrusion heat in hydrophobic microporous materials by high pressure calorimetry. Microporous and Mesoporous Materials, 134(1-3), 815.

Kirkwood, J., G., Buff, F., P. (1949) The Statistical Mechanical Theory of Surface Tension. J. of Chemical Physics. 17(3), 338-343.

Kong, X., Qiao, Y. (2005). Thermal effects on pressureinduced infiltration of a nanoporous system. Philosophical Magazine Letters, 85(7), 331 - 337.

Laouir, A., Luo, L., Tondeur, D., Cachot, T., Le Goff, P. (2003). Thermal Machines Based on Surface Energy of Wetting: Thermodynamic Analysis. AIChE Journal, 49(3), 764-781.

Laouir, A., Tondeur, D. (2008). Some New Results From Applying Thermodynamics to Wetting Phenomena. Int J. of Thermodynamics, 11(2), 61-69.

Liu, L., Zhao, J., Culligan, P., J., Qiao, Y., and Chen, X. (2009). Thermally Responsive Fluid Behaviors in Hydrophobic Nanopores. Langmuir, 25(19), 11862 11868.
Martin, T., Lefevre, B., Brunel, D., Galarneau, A., Di Renzo, F., Fajula, F., Gobin, P., et al. (2002). Dissipative water intrusion in hydrophobic MCM-41 type materials. Chemical Communications, Nol, 24-25.

Naidich, Y. V. (1972). Contact phenomena in metallic melts (in Russian). Kiev: Naukova dumka.

Qiao, Y., Punyamurtula, V., K., and Han, A. (2006). Temperature dependence of working pressure of a nanoporous liquid spring. Applied Physics Letters, 89(25), 251905.

Rusanov, A., I. (1981). The connection between the heat of evaporation and surface energy (in Russian). Reports of the USSR Academy of Sciences. 261(3), 700-703.

Suciu, C.,V., Iwatsubo, T. and Deki, Sh. (2003) Investigation of a colloidal damper. J. of Colloid and Interface Science,259(1), 62-80.

Wargaftik, N. B. (1972). Handbook of thermophysical properties of gases and liquids (in Russian). Moscow: Nauka.

Washburn, E., W. (1921). The Dynamics of Capillary Flow. The Physical Review, 17(3), 273-283.

Woo, H.-J., Monson, P., A. (2003). Phase behavior and dynamics of fluids in mesoporous glasses. The Physical Review E, 67(4), 041207. 